\title{
THE EXPERIMENTAL PERFORMANCE OF A NONLINEAR DYNAMIC VIBRATION ABSORBER
}

\author{
Yung-Sheng Hsu, Neil S Ferguson \\ Institute of Sound and Vibration Research, University of Southampton \\ Southampton, SO17 1BJ, United Kingdom \\ Email: yh1a08@,soton.ac.uk, nsf@isvr.soton.ac.uk \\ Michael J.Brennan \\ Departamento de EngenhariaMecanica, Universidade Estadual Paulista, \\ Ilha Solteira, 15385-000 Sao Paulo, Brazil \\ Email: mjbrennan@dem.feis.unesp
}

\begin{abstract}
This paper investigates the physical behaviour and effectiveness of a nonlinear dynamic vibration absorber (NDVA). The nonlinear absorber considered involves a nonlinear hardening spring which was designed and attached to a cantilever beam excited by a shaker. The cantilever beam can be considered at low frequencies as a linear single degree-of-freedom system. The nonlinear attachment is designed to behave as a hardening Duffing oscillator. The nonlinearity of the attachment is due to the particular geometrical configuration undergoing a large amplitude response. The experiment investigated the potential for vibration reduction of the system. Analytical and numerical results are presented and compared. From the measured results it was observed that the NDVA had a much wider effective bandwidth compared to a linear absorber. The frequency response curve of the NDVA has the effect of moving the second resonant peak to a higher frequency away from the tuned frequency so that the device is robust to mistuning.
\end{abstract}

Keywords: Nonlinear vibration, vibration absorber, vibration reduction, passive vibration control, Duffing oscillator

\section{INTRODUCTION}

The passive vibration absorber is an important device used for vibration reduction in structures. The linear vibration absorber is limited in that it reduces vibration over a very narrow frequency range. This range is not enough to correspond to changes in speed for a rotating unbalanced source due to load, motor power supply or source variations. A technical benefit of the NDVA has been hypothesized that they can operate efficiently over a broader range of forcing frequencies.

The bandwidth problem was first identified by Roberson [1] who considered the impractical case of an undamped absorber comprising a linear plus cubic spring acting in parallel.. Hunt and Nissen [2] presented a NDVA with a softening spring composed of a stack of Belleville washers to overcome the previous design feasibility. Nissen et al. [3] studied the optimal parameters of a NDVA and considered the technical aspects for realization. Soom [4] and Jordanov [5] have investigated both the optimal parameter design of linear and nonlinear dynamic vibration absorbers for damped primary systems. They investigated optimization criteria other than traditional measures and obtained a small improvement in the steady state response by using nonlinear springs. Zhu et al. [6] studied the system with nonlinear damping and nonlinear springs, they found that a reduction of the vibration amplitude can be obtained by adjusting the parameters of the nonlinear dampers, nonlinear spring stiffness and excitation frequency. It has also been demonstrated [7-10] that the primary phenomena behind the energy pumping produced is due to resonant interactions between coupled linear and nonlinear components.

From the review of the existing literature, it can be seen that experimental results for the nonlinear absorber have not been widely published. The aim of this paper is to partially fill this gap. The problem is first studied here analytically, to determine the most important features, before an experimental design is presented, implemented and its behavior is discussed.

\section{EQUATIONS OF MOTION OF A SINGLE DEGREE OF FREEDOM SYSTEM WITH AN ATTACHED NONLINEAR ABSORBER}

As shown in Figure 1, a NDVA is attached to a linear single degree-of-freedom main structural system. In the figure, $k_{s}, c_{s}$ and $m_{s}$ are the spring constant, viscous damping coefficient and mass of the main structural 
system respectively. For the NDVA, it has a mass $m$, a viscous damper $c$ and a nonlinear spring with a nonlinear restoring force given by the function $f(z)=k_{1} z+k_{3} z^{3}$, where $z$ is the static displacement across the spring, which has linear and nonlinear stiffness terms $k_{1}$ and $k_{3}$, respectively. The sign of $k_{3}$ denotes the nonlinear stiffness behaviour; a positive value means that the system is hardening. $x_{s}, x, \dot{x}_{s}, \dot{x}, \ddot{x}_{s}$ and $\ddot{x}$ are the displacement, velocity and acceleration of the main structural system and NDVA, respectively.

The equations of motion for this system are given by

$$
\begin{aligned}
m_{s} \ddot{x}_{s}+c_{s} \dot{x}_{s}+k_{s} x_{s}+c\left(\dot{x}_{s}-\dot{x}\right) & +k_{1}\left(x_{s}-x\right) \\
& +k_{3}\left(x_{s}-x\right)^{3}=F \cos (\omega t) \\
m \ddot{x}-c\left(\dot{x}_{s}-\dot{x}\right)-k_{1}\left(x_{s}-x\right)- & k_{3}\left(x_{s}-x\right)^{3}=0
\end{aligned}
$$

where $F$ is the amplitude of the excitation, which is periodic in time $t$ with frequency $\omega$.

It is convenient to write equations $(1 \mathrm{a}, \mathrm{b})$ in nondimensional form as

$$
\begin{aligned}
& (1+\mu) y_{s}^{\prime \prime}+2 \zeta_{s} y_{s}^{\prime}+y_{s}-\mu w^{\prime \prime}=\cos (\Omega \tau) \\
& w^{\prime \prime}+2 \zeta \omega_{0} w^{\prime}+\omega_{0}^{2} w+\gamma w^{3}=y_{s}^{\prime \prime}
\end{aligned}
$$

where the non-dimensional parameters are given by $y_{s}^{\prime \prime}=\ddot{x}_{s} / \omega_{s}^{2} x_{0}, y_{s}^{\prime}=\dot{x}_{s} / \omega_{s} x_{0}, y_{s}=x_{s} / x_{0} ; w^{\prime \prime}=\ddot{z} / \omega_{s}^{2} x_{0}$, $w^{\prime}=\dot{z} / \omega_{s} x_{0} \quad, \quad w=z / x_{0}, \mu=m / m_{s}, \gamma=\left(k_{3} / \mu k_{s}\right) x_{0}^{2}$, $\zeta_{s}=c_{s} / 2 m_{s} \omega_{s}, \zeta=c / 2 m \omega_{1}, \omega_{0}=\omega_{1} / \omega_{s}$ and $\Omega=\omega / \omega_{s}$, where $\omega_{s}^{2}=k_{s} / m_{s}, \omega_{1}^{2}=k_{1} / m$ and $x_{0}$ is the static extension of the linear spring due to a static force of amplitude $F$, and $(\bullet)^{\prime}=\mathrm{d}(\bullet) / d \tau$ in which $\tau=\omega_{s} t$ is non-dimensional time.

The fundamental assumption in the Harmonic Balance method (HBM) approach used for the first order solution is that the response of the main system and the absorber is predominantly harmonic at the excitation frequency. Applying the HBM, it is assumed that a solution is of the form

$$
\begin{aligned}
& y_{s}=Y_{s} \cos \left(\Omega \tau+\varphi_{s}\right) \\
& w=W \cos (\Omega \tau+\varphi)
\end{aligned}
$$

Substituting equations $(3 a, b)$ into equations $(2 a, b)$ gives two expressions involving the amplitudes of the responses of the two masses, namely

$$
\begin{aligned}
& Y_{s}^{2}=\frac{1}{\Omega^{4}}\left(\begin{array}{l}
\frac{9}{16} \gamma^{2} W^{6}+\frac{3}{2} \gamma\left(\omega_{0}^{2}-\Omega^{2}\right) W^{4} \\
+\left(\Omega^{4}+4 \zeta^{2} \Omega^{2} \omega_{0}^{2}+\omega_{0}^{4}-2 \Omega^{2} \omega_{0}^{2}\right) W^{2}
\end{array}\right) \\
& a W^{6}+b W^{4}+c W^{2}+d=0 \\
& \text { where } \\
& a=\frac{9}{16} \gamma^{2}, b=\frac{3}{2} \gamma\left(\omega_{0}^{2}-\Omega^{2}\right)+\Omega^{4} \frac{g}{e},
\end{aligned}
$$

$$
\begin{aligned}
& c=\left(\omega_{0}^{2}-\Omega^{2}\right)^{2}+4 \zeta^{2} \omega_{0}^{2} \Omega^{2}+\Omega^{4} \frac{h}{e}, d=-\frac{\Omega^{4}}{e}, \\
& e=\left(1-\Omega^{2}(1+\mu)\right)^{2}+4 \zeta_{s}^{2} \Omega^{2}, g=-\frac{3}{2} \gamma \mu\left(1-\Omega^{2}(1+\mu)\right), \\
& h=\Omega^{4} \mu^{2}-2 \mu\left(1-\Omega^{2}(1+\mu)\right)\left(\omega_{0}^{2}-\Omega^{2}\right)+8 \zeta_{s} \zeta \mu \omega_{0} \Omega^{2}
\end{aligned}
$$

Once the parameters for the system and the excitation frequency have been specified, then the solutions of equation (4b) in $W^{2}$ give the three numerical solutions, which should be checked for physical interpretation and existence. It is noted that only real solutions for $W^{2}$ are physical responses. When the normalised relative displacement $W$ has been determined it can be substituted into equations (4a) to obtain the amplitude of the main system $Y_{s}$.

Three steady-state solutions of equation (4b) are given by $W_{1}^{2}=s+t-\frac{b}{3 a}$,

$W_{2,3}^{2}=-\frac{1}{2}(s+t)-\frac{b}{3 a} \pm j \frac{\sqrt{3}}{2}(s-t)$

where

$$
s=\sqrt[3]{r+\sqrt{\frac{-\Delta}{108 a^{4}}}}, t=\sqrt[3]{r-\sqrt{\frac{-\Delta}{108 a^{4}}}}, r=\frac{9 a b c-27 a^{2} d-2 b^{3}}{54 a^{3}},
$$$$
\Delta=18 a b c d-27 a^{2} d^{2}-4 b^{3} d+b^{2} c^{2}-4 c^{3} a
$$

Depending on the degree of nonlinearity in the NDVA system, there are combinations of the parameters which produce a multivalued response. To find the conditions for such a response, $\Delta$, the discriminant of the cubic polynomial in $W^{2}$ in Equation (4a) can be examined [11]. If $\Delta>0$ there are three distinct real roots, if $\Delta<0$ there is one real root and a pair of complex conjugate roots and if $\Delta=0$ then there are at least two real coincident roots.

\section{VIBRATION REDUCTION OF A NDVA}

The influence of the nonlinear stiffness parameter $(\gamma)$ and the damping ratio $(\zeta)$ on the reduction of vibration can now be investigated analytically. These were also checked by direct numerical integration (using the MATLAB ode45 function) of the equations of motion. The combined system response is nonlinear and is compared in terms of the relative response to the amplitude of the static response of the primary system on its own when an equal static force is applied.

\subsection{Influence of the nonlinear parameter $(\gamma)$}

In Figures 2(a)-(b), the effect on the normalized primary system displacement $Y_{s}$ can be seen. It is to shift the first resonance peak $\Omega_{r 1}$ and bend the second resonance peak $\Omega_{r 2}$ to the right. An unstable branch appears in between the two stable branches of the first resonance peak at the frequency $\Omega_{r 1}$. For low values of 
the nonlinearity, the unstable branch may appear at a response level above the stable branches of the second resonance peak. For higher values of the nonlinearity, the two stable branches intersect each other and the amplitude of the unstable branch is above the two stable branches.

The vibration reduction band can be observed in Figures 2(a)-(b), i.e., bandwidth where $Y_{s}=X_{s} / X_{0} \leq 1$, which contains the effective tuned frequency. The difference is apparent between the vibration reduction of the linear and the nonlinear absorbers; the nonlinear device has a much wider effective bandwidth. It is increased by about $6 \%$ and $211 \%$ compared to the linear case for values of the nonlinear stiffness parameter of $\gamma=10^{-5}$ and $\gamma=10^{-4}$ respectively. These were also checked by numerical integration of the equations of motion. Equations (1a,b) were solved numerically and the Fourier coefficients extracted from the time histories. The amplitude of the first of these coefficients is depicted by circles in the frequency response curves shown here. It is noted that in order to find the stable multivalued responses the initial conditions for the displacement and velocity need to be adjusted.

Figures 2(a)-(b) also shows that the first resonance frequency of the system with the nonlinear absorber $\Omega_{r 1}$ moves to a slightly higher frequency as it is affected by the nonlinearity. The peak response of the primary mass at the first resonance frequency is also higher than that for the linear absorber case.

\subsection{The effect of damping $(\zeta)$ in the nonlinear absorber}

In Figures 3(a)-(b) the linear damping ratio for the attached system is increased from $\zeta=2 \times 10^{-3}$ to $\zeta=8 \times 10^{-3}$. The difference between the vibration response due to the linear and the nonlinear absorber in Figures 3(a)-(b) is again that the nonlinear absorber has a much wider bandwidth. It is increased by about $211 \%$ and $255 \%$ compared to the linear case for a value of $\zeta=2 \times 10^{-3}$ and $\zeta=8 \times 10^{-3}$, respectively. It is noted that for a larger damping ratio $(\zeta)$, the wider the reduction bandwidth of the nonlinear absorber compared to the linear case and the reduction at the effective tuned frequency is less. As $\zeta$ is increased in the nonlinear absorber case, the vibration reduction bandwidth decreases. In addition, in the response curves of the system, corresponding to larger damping, at the second resonance frequency $\Omega_{r 2}$ of the system with the nonlinear absorber will shift to a lower frequency. The amplitude of the first resonance frequency $\Omega_{r 1}$ of the system slightly reduces.

By adding damping, the displacement of the primary system can be made to be single valued for all frequencies, so that no jumps can occur in the response. A disadvantage though is that higher damping results in less vibration reduction at the tuned frequency $\Omega_{\text {. }}$. For the case of high damping, one might expect that the nonlinear absorber will dissipate more vibration energy at some frequencies when the relative velocity is high. Thus, the 'resonance' peaks might be expected to be significantly reduced with increased damping. This is apparent in the reduction in the peak response amplitude at the resonance frequencies $\Omega_{r 1}, \Omega_{r 2}$ for the linear absorber, but it does not appear to be the case for the nonlinear absorber.

\section{EXPERIMENTAL VALIDATION}

A nonlinear absorber was designed and attached to a cantilever beam which was excited by a shaker. The overall system is modelled as a nonlinear hardening Duffing oscillator coupled to a linear system. The aim of the experimental investigation is to demonstrate the corresponding vibration reduction of the configuration using this particular nonlinear vibration absorber.

\subsection{Nonlinear dynamic vibration absorber design and experimental investigation}

The phenomenon of nonlinear stiffness was reproduced using a thin clamped circular plate undergoing large flexural deflection [12]. The absorber mass was attached at the centre of the thin circular plate. The plate is clamped by a frame on its edges as illustrated in Figure 4. The circular plate has a radius $r$, thickness $h$, Poisson's ratio $v$ and Young's modulus $E$. When the mass moves in the vertical direction, the plate bends with a large deflection producing axial strain and a change in length of the midplane axis. This large deflection, producing geometric nonlinearity, is the cause of the nonlinearity in the restoring force and hence effective stiffness of the absorber. The static relationship between applied static force $f$ at the centre of the circular plate and the deflection at that point has been obtained when $v$ is equal to 0.3 [12]

$0.217 \frac{f r^{2}}{E h^{4}}=\frac{y}{h}+0.443\left(\frac{y}{h}\right)^{3}$

It can be written as

$f=k_{1} y+k_{3} y^{3}$

where $k_{1}=\frac{E h^{3}}{0.217 r^{2}}$ and $k_{3}=\frac{0.443 E h}{0.217 r^{2}}$ are the corresponding stiffness coefficients.

The practical implementation of the nonlinear vibration absorber is shown in Figures 5-7. Photographs are shown in Figure 5-6and a schematic representation is shown in Figure 7. A mass $m$ was attached to the thin plate, which is itself bolted to a cantilever beam by a support frame. 
The spring characteristics between the absorber mass and the support frame are due to the thin circular plate, which can be modelled as a nonlinear stiffness $k_{1}, k_{3}$ and for small dissipation effects a viscous damper $c$ was introduced. The thickness of the plate or shim and the attached mass can be altered and these have a large effect on the nonlinear attachment system characteristics. In addition, the length of the cantilever beam can also be altered, so it is possible to consider different natural frequencies. For large dynamic deformations it was hypothesized that the absorber would be nonlinear. The cantilever beam was excited by an electro-dynamic shaker. The support frame and beam structure without the absorber can be modelled as a linear system comprising of a spring $k_{s}$, a viscous damper $c_{s}$ and a mass $m_{s}$. Applying a constant amplitude force at each frequency to the shaker, the excitation can be modelled as a constant amplitude harmonic force, as shown in Figure 7.

\subsection{Experimental procedure and results}

The schematic diagram of the experimental setup is shown in Figure 8. The electro-dynamic shaker was driven by a signal generator producing a stepped-sine signal. The accelerometers (PCB type 352C22) were attached to the support structure and to the mass of the absorber, while the oscilloscope was used to observe the system response.

A preliminary test was implemented to broadly investigate the dynamic behaviour of the system. For each test, the shaker had a different force amplitude. In the high force amplitude test, a slow frequency sweep (where the force gauge recorded a constant voltage and hence force amplitude) was applied from $100 \mathrm{~Hz}$ to about $225 \mathrm{~Hz}$ and the response of the system was observed using the oscilloscope. The first resonance was monitored at around $123 \mathrm{~Hz}$, with large vibrational amplitudes in both systems. In addition, the first resonance peak was difficult to measure because of the light damping in the cantilever beam. When the frequency was increased beyond this, the tuned frequency was observed at about $183 \mathrm{~Hz}$. In this frequency region the vibration of the support frame was a minima. However, a second resonance occurred at about $213 \mathrm{~Hz}$, where only the vibration of the absorber mass was large. This was followed by a sudden decrease in the motion of the mass of absorber; a jump-down in the response. The frequency was then slowly swept down from this high frequency back to the low frequency. A sudden increase in the amplitude was observed at a frequency of about $199 \mathrm{~Hz}$, again only for the mass of the absorber (a jump-up). At $123 \mathrm{~Hz}$, the resonance response, for which there was large motion of both the support structure and the mass of absorber, was observable. In the low force amplitude test, the response behaviour was observed that is approximately similar to the a linear system. The first resonance, tuned frequency and second resonance were found to occur at around $125 \mathrm{~Hz}, 160 \mathrm{~Hz}$ and $172 \mathrm{~Hz}$ respectively. The jump-up and jump-down frequencies did not occur.

For the measured data presented, the shaker was driven at discrete frequencies for the system with the thin plate, corresponding to the cases described above. The excitation frequency was increased from $100 \mathrm{~Hz}$ to 225 $\mathrm{Hz}$, in $1 \mathrm{~Hz}$ increments, and then decreased to $100 \mathrm{~Hz}$ with the same frequency decrements. The amplitude of the excitation force was maintained at a constant level for all excitation frequencies, by manually adjusting the power amplifier so that the output voltage of the force gauge was $127 \mathrm{mV}$ and $12.7 \mathrm{mV}$ respectively. This corresponded to an equivalent force of $1.12 \mathrm{~N}$ and $0.11 \mathrm{~N}$ respectively. At each frequency, once the system was at steady-state, five seconds of acceleration time histories were captured using a DataPhysics frequency analyser connected to a PC. Subsequently, the acceleration of the support frame and beam structure and the absorber were measured, and then this data was processed to give the displacement. The data is presented in terms of the absolute displacement $x_{s}$ of the support frame and beam structure and the absolute displacement $x$ of absorber. The Fourier series coefficients were extracted from these time histories and the amplitude of the first harmonic of each data set is plotted at the corresponding excitation frequency. This can be seen in Figures 9(a)-(b) for the system for which the forces have low and high amplitudes respectively. At low force amplitude, the data points in each graph are dashed-dotted line. At high force amplitude, the data points in each graph are denoted by ' + ' for increasing frequency and ' $\bullet$ ' for decreasing frequency respectively.

The response of the support frame and beam structure $X_{s}$ is plotted in Figure 9(a). It can be seen that the first resonance frequency and tuned frequency occur at about $125 \mathrm{~Hz}, 160 \mathrm{~Hz}$ and $123 \mathrm{~Hz}, 183 \mathrm{~Hz}$ for the low and high force amplitude cases, respectively. The nonlinear system attached to the cantilever beam structure has a great effect on its response. In addition, the jump-down frequency occurs at approximately $213 \mathrm{~Hz}$ and the corresponding jump-up frequency at about $199 \mathrm{~Hz}$ for the high force amplitude. However, the jump-up and jump-down frequencies did not occur for the low force amplitude. In Figure 9(b), which shows the response of the absorber $X$, in addition to the peak associated with the first resonance frequency of the support frame and beam structure, a jump-down and a jump-up frequency can also be observed for the high force amplitude.

\subsubsection{Parameter estimation and model validation}

The cantilever beam was made of aluminium with a

total length $L=0.09 \mathrm{~m}, \quad$ cross-sectional area $A=0.04 \mathrm{~m} \times 0.004 \mathrm{~m}$, density $\rho=2700 \mathrm{~kg} / \mathrm{m}^{3}$, and Young's modulus $E=70 \mathrm{GN} / \mathrm{m}^{2}$. In addition, the 
circular plate was made of brass with thickness $0.2 \times 10^{-3} \mathrm{~m}$, area $A=\pi(0.026)^{2} \mathrm{~m}^{2}$, density $\rho=8500 \mathrm{~kg} / \mathrm{m}^{3}$, and Young's modulus $E=110 \mathrm{GN} / \mathrm{m}^{2}$. The parameters for the systems tested were required in order to compare the experimental results with the model predictions. These parameters $\left(m_{s}, c_{s}, k_{s}, m, c, k_{1}, k_{3}\right)$ were measured independently and were estimated as follows.

The Frequency Response Function (FRF) of the support frame attached to the cantilever beam without the absorber was measured using pseudo random force measurements. The system parameters (mass $m_{s}$, damping $c_{s}$ and stiffness $k_{s}$ ) were estimated by fitting a theoretical single degree of freedom FRF to the experimental FRF. In addition, the mass of the absorber $m$ was measured directly. The viscous damping coefficient $c$ of the nonlinear attachment was estimated on it separately through the half power points method at low amplitude [13]. Moreover, the stiffnesses $k_{1}$ and $k_{3}$ of the nonlinear attachment were estimated using the measurements of the static displacement for applied static loads. Above these parameters are listed in Table 1. The equivalent system parameters for the equation of motion written in the non-dimensional form of Equations (2a,b) are listed in Table 2. It is noted that the system was designed such that by simply adjusting the thickness of the plate, in the vibration absorber, the nonlinear stiffness and natural frequency of the absorber could be varied.

The frequency bandwidth was determined such that $X_{s} \leq X_{0}$ in that frequency range, where $X_{0}$ is the corresponding static extension of the linear spring represented by the cantilever beam for the same magnitude of the static load. The response $X_{s}=X_{0}$ is the thin solid horizontal line shown in Figures 9-10(a). Examining Figure 9-10(a), the high force amplitude applied for nonlinear absorber has a much wider vibration reduction bandwidth. The numerical parameters for the system are given in Table 2. The frequency response curves described by Equations $(4 a, b)$ are shown in Figure 10. In Table 3, for the approximate HBM solution, the bandwidth for vibration reduction is increased by about $311 \%$ and $188 \%$ for the increasing and decreasing frequency cases, respectively. For the measurement, it is increased by about $280 \%$ and $240 \%$ for the increasing and decreasing frequency cases, respectively.

\section{CONCLUSIONS}

This paper has investigated the influence of the NDVA parameters on the vibration reduction. The nonlinearity resulted in a much wider effective bandwidth compared to that for a linear absorber with similar mass and damping. It was found that the frequency response curve of the NDVA has the effect of moving the second resonant peak to a higher frequency away from the tuned frequency, so that the device is robust to mistuning. Experimental results have been presented to compare with the model derived.

\section{REFERENCES}

1. Roberson, R. E. (1952). "Synthesis of a nonlinear dynamic vibration absorber." Journal of the Franklin Institute 254(3): 205-220.

2. Hunt, J. B. and Nissen, J. C. (1982). "The broadband dynamic vibration absorber." Journal of Sound and Vibration 83(4): 573-578.

3. Nissen, J. C., Popp, K., et al. (1985). "Optimization of a non-linear dynamic vibration absorber." Journal of Sound and Vibration 99(1): 149-154.

4. Soom, A. and Lee, M. (1983). "Optimal design of linear and non-linear vibration absorbers for damped systems." Trans ASME: Journal of vibration, acoustics, stress, and reliability in design 105: 112-119.

5. Jordanov, I. N. and Cheshankov, B. I. (1988). "Optimal design of linear and non-linear dynamic vibration absorbers." Journal of Sound and Vibration 123(1): 157170.

6. Zhu, S. J., Zheng, Y. F., et al. (2004). "Analysis of nonlinear dynamics of a two-degree-of-freedom vibration system with non-linear damping and non-linear spring." Journal of Sound and Vibration 271(1-2): 15-24.

7. Gendelman, O. V., Gourdon, E., et al. (2006). "Quasiperiodic energy pumping in coupled oscillators under periodic forcing." Journal of Sound and Vibration 294(4-5): 651-662.

8. Gourdon, E., Alexander, N. A., et al. (2007). "Nonlinear energy pumping under transient forcing with strongly nonlinear coupling: Theoretical and experimental results." Journal of Sound and Vibration 300(3-5): 522-551.

9. Gendelman, O., Starosvetsky, Y., et al. (2008). "Attractors of harmonically forced linear oscillator with attached nonlinear energy sink I: Description of response regimes." Nonlinear Dynamics 51(1): 31-46.

10. Starosvetsky, Y. and Gendelman, O. (2008). "Attractors of harmonically forced linear oscillator with attached nonlinear energy sink. II: Optimization of a nonlinear vibration absorber." Nonlinear Dynamics 51(1): 47-57.

11. 2008, http://en.wikipedia.org/wiki/Cubic equation.htm 12. S. Timoshenko, S. W.-K. (1959). "Theory of plates and shells", New York, McGraw-Hill: 580.

13. Mead, D. J. (1998). "Passive vibration control", New York, Wiley. 


\begin{tabular}{|c|c|c|c|c|}
\hline & $m_{s}(\mathrm{~kg})$ & $\begin{array}{c}c_{s} \\
(\mathrm{~N} \cdot \mathrm{s} / \mathrm{m})\end{array}$ & $k_{s}(\mathrm{~N} / \mathrm{m})$ & $F(\mathrm{~N})$ \\
\hline \multirow{2}{*}{$\begin{array}{l}\text { Low force } \\
\text { amplitude } \\
\text { High force } \\
\text { amplitude }\end{array}$} & \multirow[t]{2}{*}{$126 \times 10^{-3}$} & \multirow[t]{2}{*}{1.26} & \multirow[t]{2}{*}{$8.93 \times 10^{4}$} & 0.11 \\
\hline & & & & 1.12 \\
\hline & $m(\mathrm{~kg})$ & $\begin{array}{c}c \\
(\mathrm{~N} \cdot \mathrm{s} / \mathrm{m})\end{array}$ & $k_{1}(\mathrm{~N} / \mathrm{m})$ & $k_{3}\left(\mathrm{~N} / \mathrm{m}^{3}\right)$ \\
\hline $\begin{array}{l}\text { Low force } \\
\text { amplitude } \\
\text { High force } \\
\text { amplitude }\end{array}$ & $6.44 \times 10^{-3}$ & 0.15 & $5.69 \times 10^{3}$ & $6.90 \times 10^{10}$ \\
\hline
\end{tabular}

Table 1 The estimated system parameters from the experimental tests.

\begin{tabular}{|l|c|c|c|c|c|}
\hline & $\mu$ & $\omega_{0}$ & $\gamma$ & $\zeta_{s}$ & $\zeta$ \\
\hline $\begin{array}{l}\text { Low force } \\
\text { amplitude }\end{array}$ & $5.11 \times 10^{-2}$ & 1.17 & $2.29 \times 10^{-5}$ & $5.95 \times 10^{-3}$ & $1.18 \times 10^{-2}$ \\
\cline { 1 - 1 } $\begin{array}{l}\text { High force } \\
\text { amplitude }\end{array}$ & & & $2.37 \times 10^{-3}$ & & \\
\hline
\end{tabular}

Table 2 Equivalent non-dimensional system parameters for the model predictions.

\begin{tabular}{|c|c|c|}
\hline HBM solution & $\begin{array}{l}\text { Low force } \\
\text { amplitude }\end{array}$ & $\begin{array}{l}\text { High force } \\
\text { amplitude }\end{array}$ \\
\hline $\begin{array}{c}\text { Bandwidth for } \\
\text { increasing frequency }\end{array}$ & 9 & 37 \\
\hline Bandwidth improvement (\%) & & 311 \\
\hline $\begin{array}{c}\text { Bandwidth for } \\
\text { decreasing frequency }\end{array}$ & 9 & 28 \\
\hline Bandwidth improvement (\%) & & 188 \\
\hline Measurements & $\begin{array}{l}\text { Low force } \\
\text { amplitude }\end{array}$ & $\begin{array}{l}\text { High force } \\
\text { amplitude }\end{array}$ \\
\hline $\begin{array}{c}\text { Bandwidth for } \\
\text { increasing frequency }\end{array}$ & 10 & 38 \\
\hline Bandwidth improvement (\%) & & 280 \\
\hline $\begin{array}{c}\text { Bandwidth for } \\
\text { decreasing frequency }\end{array}$ & 10 & 34 \\
\hline Bandwidth improvement (\%) & & 240 \\
\hline
\end{tabular}

Table 3 The bandwidth frequency of NDVA on the primary system frequency response curves.

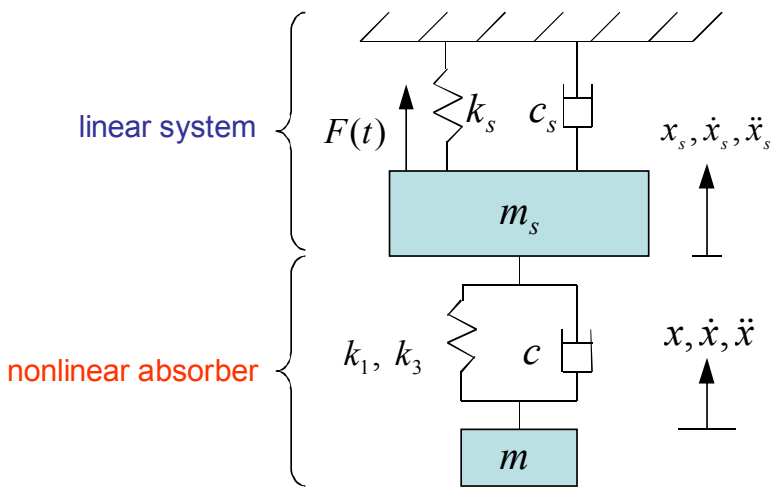

Figure 1 A nonlinear dynamic vibration absorber (NDVA) attached to a single degree-of-freedom main system.
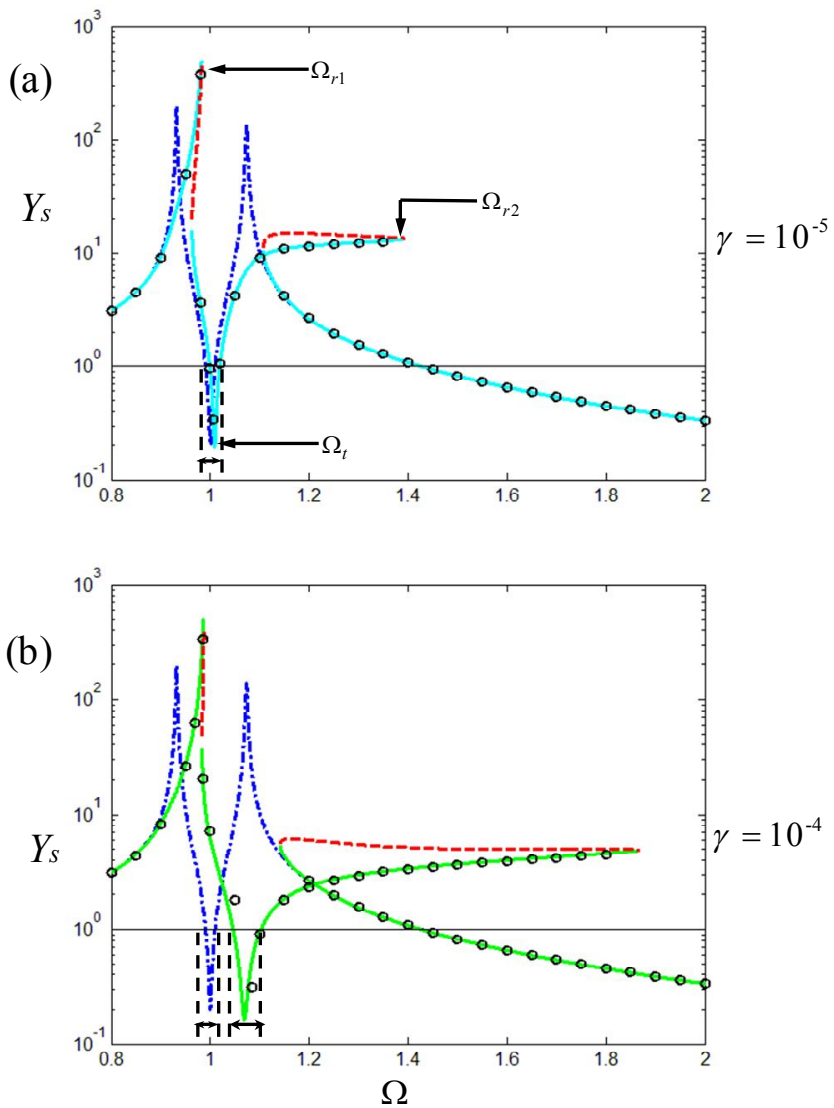

Figure 2 Plots showing the effect of the nonlinear absorber stiffness on the primary system frequency response curves $Y_{s}$ as a function of $\Omega$. (The 'tuned' frequency $\omega_{0}=\omega_{1} / \omega_{s}=1$, mass ratio $\mu=0.02$ and damping $\left.\zeta_{s}=0.001, \zeta=0.002\right)$. The response for the system with the linear absorber is given by the dasheddotted line, the solid line is the stable solution and the dashed line gives the unstable solution. Direct numerical solutions are shown by the symbol (' $\circ$ '). (a) $\gamma=10^{-5}$ low absorber stiffness and (b) $\gamma=10^{-4}$ high absorber stiffness. 

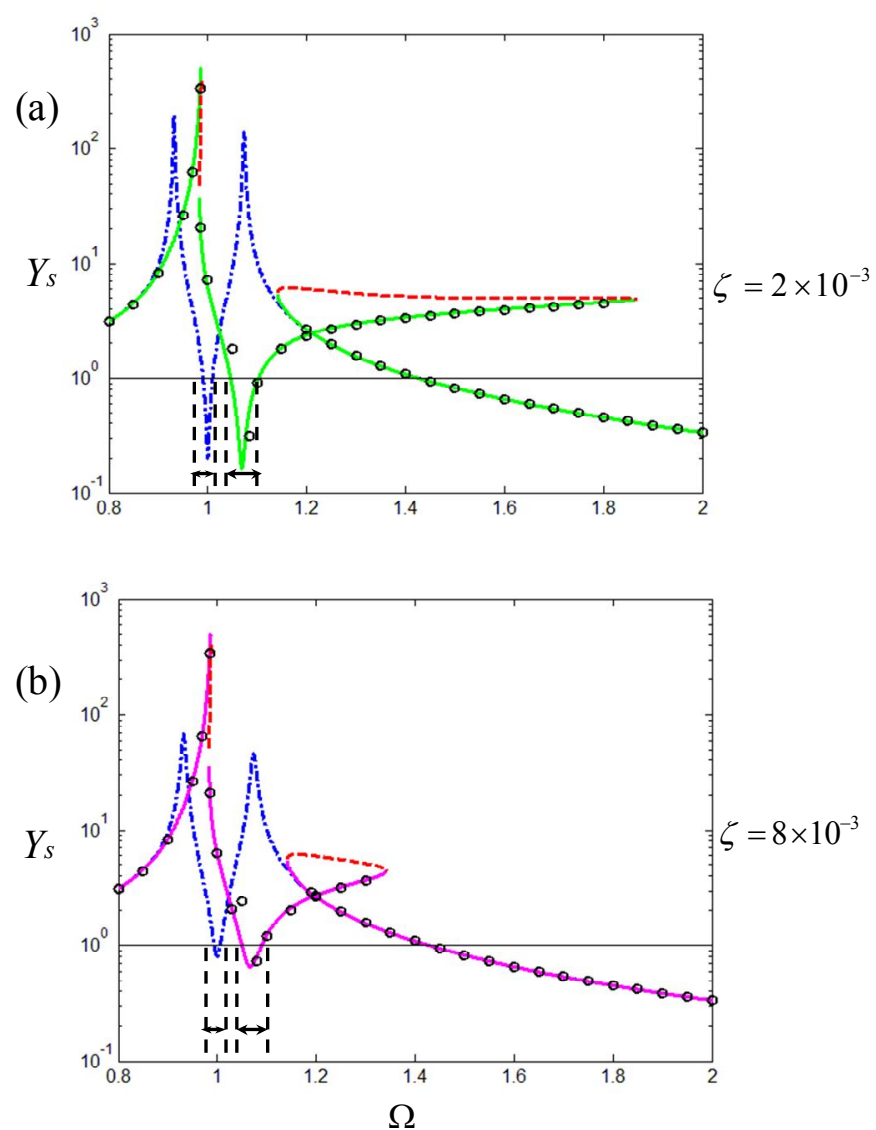

Figure 3 Plots showing the effect of the damping in the nonlinear absorber on the primary system frequency response curves $Y_{s}$ as a function of $\Omega$. (The 'tuned' frequency $\omega_{0}=\omega_{1} / \omega_{s}=1$, nonlinear absorber stiffness $\gamma=10^{-4}$, mass ratio $\mu=0.02$ and damping $\zeta_{s}=0.001$ ). The response for the system with the linear absorber is given by the dashed-dotted line, the solid line is the stable solution and the dashed line gives the unstable solution. Direct numerical solutions are shown by the symbol (' $\circ$ '). (a) $\zeta=2 \times 10^{-3}$ low absorber damping and (b) $\zeta=8 \times 10^{-3}$ high absorber damping.

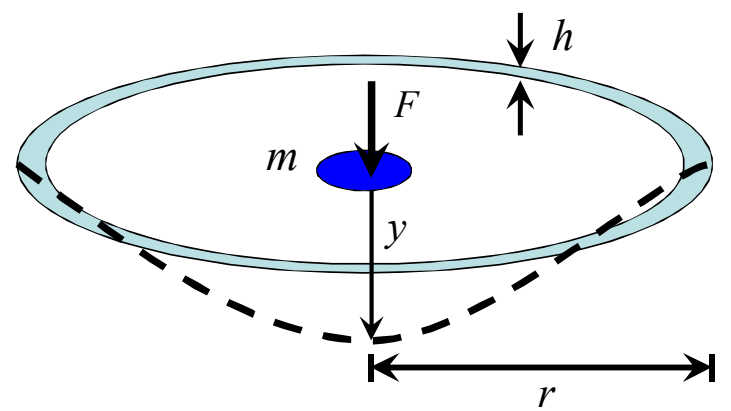

Figure 4 Schematic representation of a nonlinear vibration absorber using a thin circular plate.

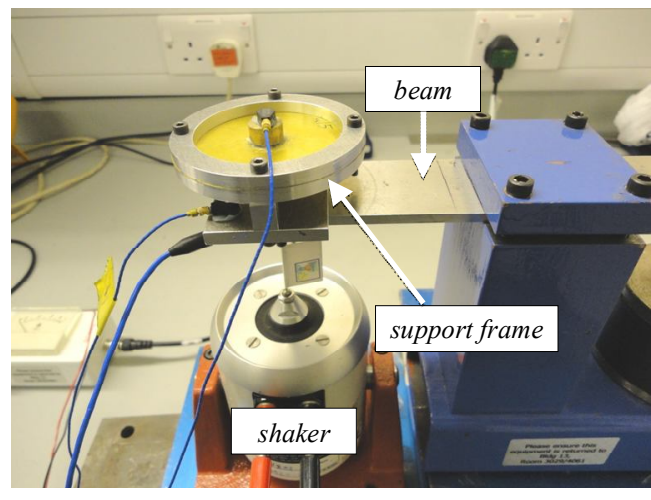

Figure 5 Photograph of the actual experimental system consisting of a nonlinear absorber attached to a cantilever beam excited by an electro-dynamic shaker.

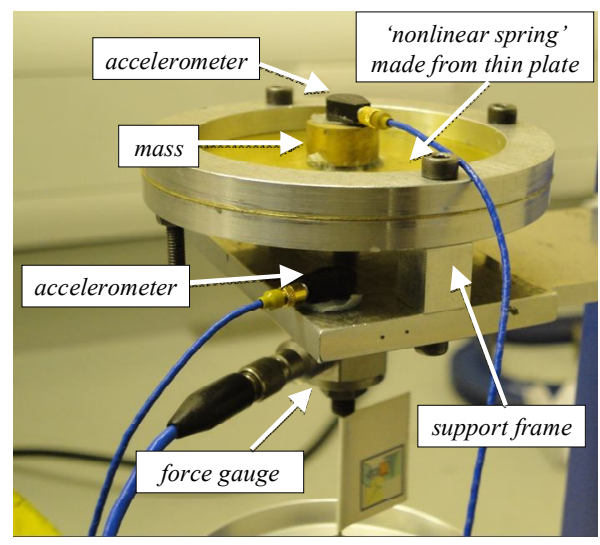

Figure 6 Photograph showing the details of the nonlinear system.

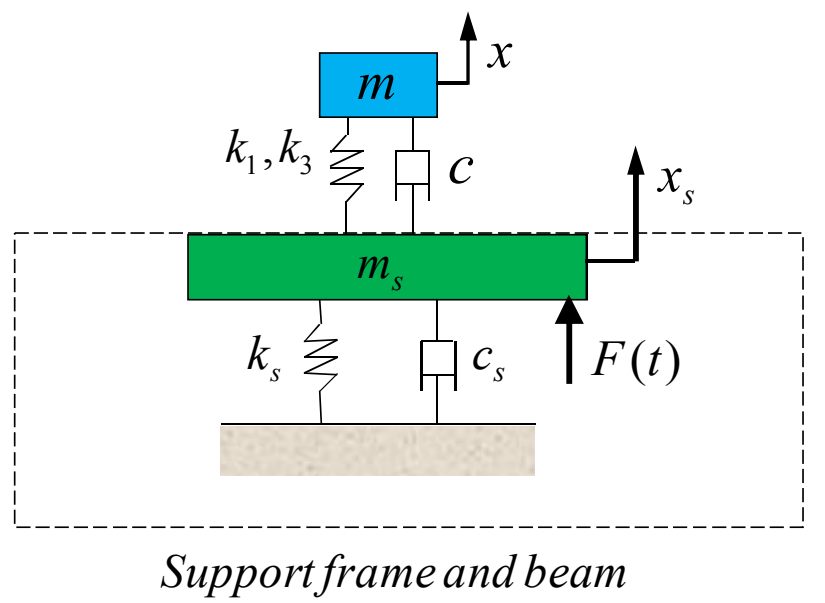

Figure 7 Schematic representation of a nonlinear absorber attached to a cantilever beam system excited by a shaker. 


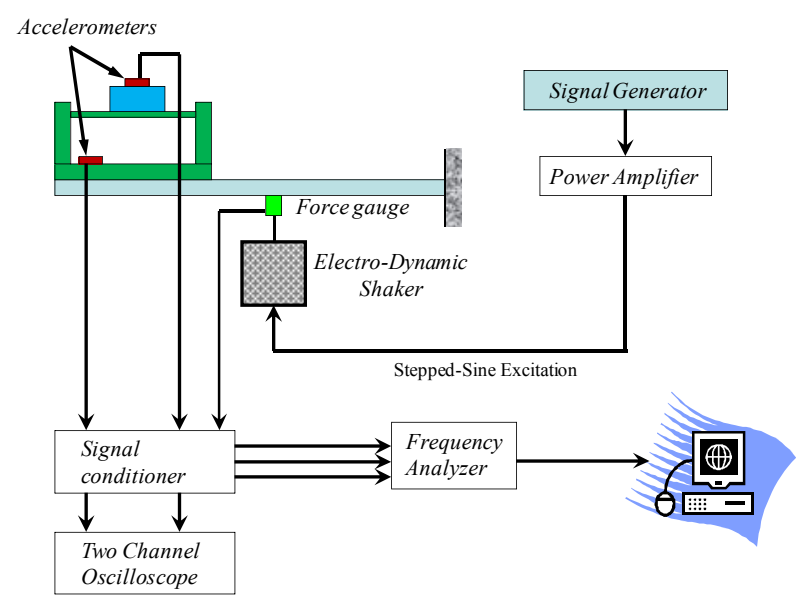

Figure 8 Schematic diagram of the instrumentation setup used for the laboratory tests under harmonic excitation.
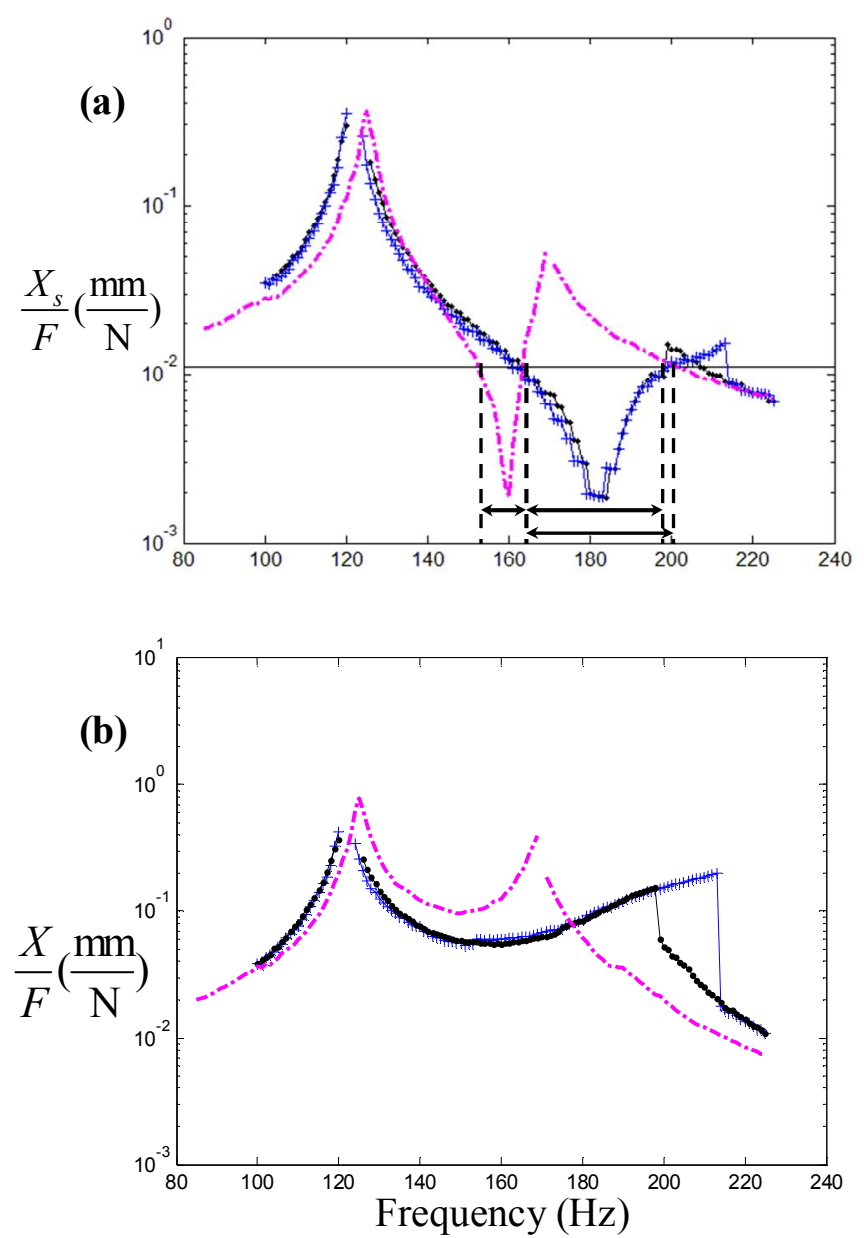

Figure 9 Comparison of the measured frequency response curves for the system in Table 2. Plate thickness 0.2 (mm): (a) Absolute displacement of the beam structure, (b) Absolute displacement of the absorber. Low force amplitude for $F=0.11 \mathrm{~N}$ (dashed-dotted line). High force amplitude for $F=1.12 \mathrm{~N}$ : increasing frequency $\left({ }^{\prime}+\right.$ ' $)$, decreasing frequency $(' \bullet ')$.
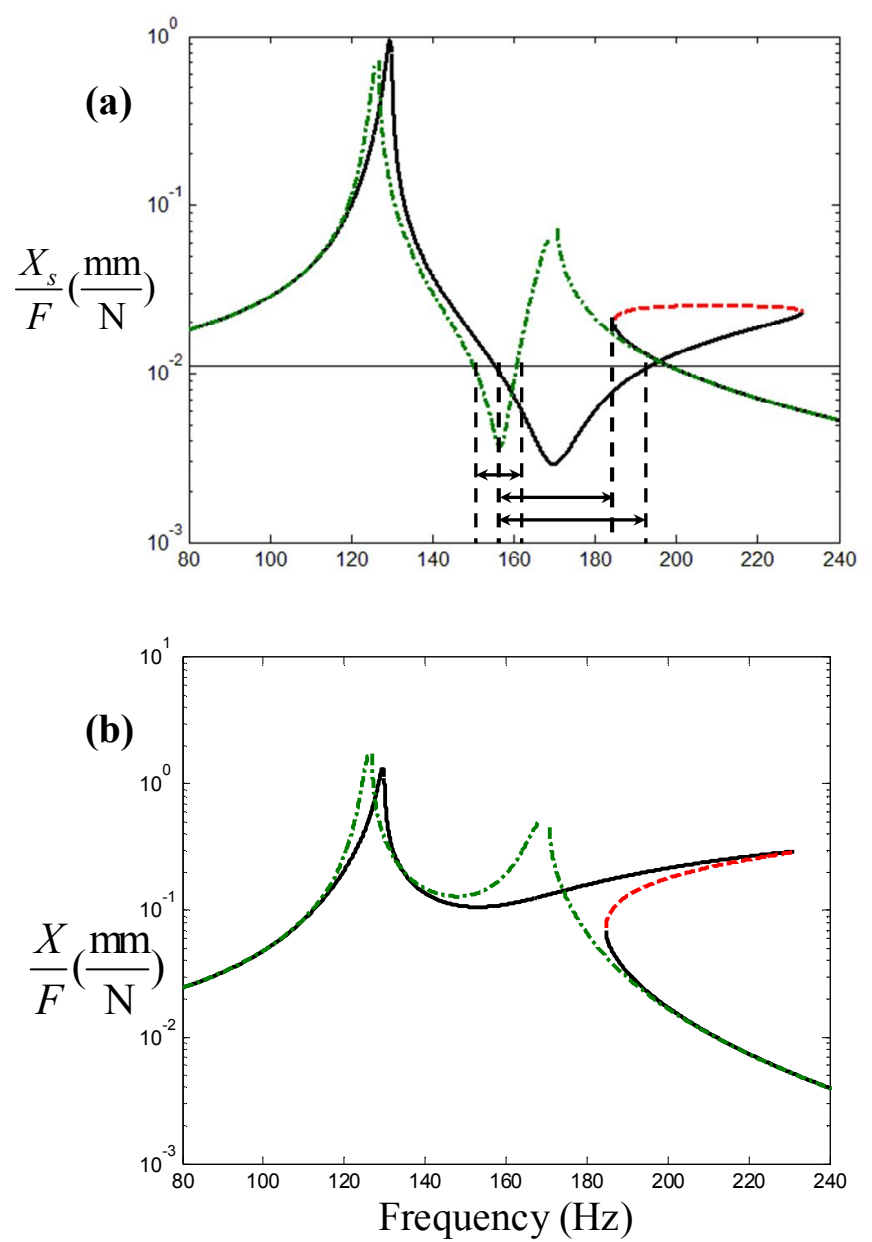

Figure 10 Comparison of the predicted frequency response curves (HBM solution) for the system in Table 2. Plate thickness $0.2(\mathrm{~mm})$ : (a) Absolute displacement of the beam structure, (b) Absolute displacement of the absorber. Low force amplitude for $F=0.11 \mathrm{~N}$ (dasheddotted line). High force amplitude for $F=1.12 \mathrm{~N}$ : stable solution (solid line), unstable solution (dashed line). 